\title{
The Effect of Meditation on Regulation of Heart Rate
}

\author{
Mohammad Karimi Moridani ${ }^{1 *}$, Pargol Yousefi ${ }^{2}$ and Anahita Safavi Sohi ${ }^{2}$ \\ Department of Biomedical Engineering, Faculty of health, Tehran Medical Sciences, Islamic Azad University, Iran \\ Department of Electrical Engineering, South Tehran Branch, Islamic Azad University, Iran
}

*Corresponding author: Mohammad Karimi Moridani, Department of Biomedical Engineering, Faculty of health, Tehran Medical Sciences, Islamic Azad University, Tehran, Iran.

To Cite This Article: Mohammad Karimi Moridani, Pargol Yousefi and Anahita Safavi Sohi, The Effect of Meditation on Regulation of Heart Rate. Am J Biomed Sci \& Res. 2021 - 12(2). AJBSR.MS.ID.001736. DOI: 10.34297/AJBSR.2021.12.001736.

Received: 眥 January 25, 2021; Published: 眥 March 16, 2021

\begin{abstract}
This study aimed to analyze the dynamics of heart rate before and during meditation to investigate the effect of meditation on heart rate regulation. For this purpose, heart rate signals from the Physiont database were used. Linear and nonlinear features were calculated in two data sets before and during meditation. The results show that the heart rate fluctuations decrease during meditation, and the heart rate rhythm becomes more regular. In contrast, before meditation, the criteria for heart rate changes and heart rate fluctuations are high. Also, the result indicates a decrease in mean heart rate during meditation. It has been shown that the suggested features can be useful in evaluating the heart rate signal during meditation.

Meditation has been considered an adjunct therapy in managing psychiatric illnesses (such as anxiety and depression) and maintaining mental health. As a result, the use of meditation can be very effective in preventing cardiovascular disease due to its positive effect on heart rhythm regulation and relaxation.
\end{abstract}

Keywords: Heart rate; Meditation; Regulation; Detection; Nonlinear features

\section{Introduction}

Each year, patients with acute coronary syndrome symptoms and myocardial infarction account for $10 \%$ of emergency cases and up to $25 \%$ of all patient admissions [1,2]. This represents a significant financial burden on health care resources, with the cost of treating these patients amounting to approximately $\$ 286$ billion annually, or negligibly $17 \%$ of health care costs [3], which emphasizes that cardiovascular interventions such as meditation are needed to help. Over the past few decades, awareness of the use of meditation or other forms of mind-body therapy in health care has increased.

Today, heart disease, especially coronary heart disease, is one of the three leading causes of death, cancer, and stroke in most industrialized countries [4]. Stress and anxiety are a significant risk factor for cardiovascular disease [5]. Psychological stress (PS) has several effects on coronary artery disease classified into behavioural and direct pathophysiological mechanisms [6]. Also, increased stress contributes to direct pathophysiological processes that increase the risk of coronary artery disease. Chronic psychological stress causes significant platelet function impairment, including increased platelet reactivity and platelet production releases, such as platelet factor 4 and Beta-thromboglobulin $[7,8]$. Besides, during stress, the risk of blood clots increases. The clot formed can block an artery, and if it is a coronary artery, this blockage can lead to a heart attack [9]. Stress also causes cardiovascular changes that are associated with coronary heart disease. For example, the blood of people with stress has high levels of activated platelets and bad cholesterol [10]. While chronic PS increases coronary artery disease risk, acute PS can cause heart attack, cardiac arrhythmia, and narrowing of the arteries [11]. Stress and anxiety can also lead to decreased heart rate, increased resting heart rate, and delayed HR recovery after exercise [12]. Also, PS causes oxidative stress 
and ultimately heart failure [13]. Thus, psychological stress and depression cause arterial abnormalities, platelet dysfunction, and increased hemodynamic stress (including recording systolic and diastolic pressure and heart rate). All of these factors increase the risk of cardiovascular attacks [14]. Research shows that meditation exercises have beneficial effects on psychological risk factors [1518]. Scientific studies from the 1960 s onwards show that meditation affects the body's metabolism, endocrine system, central nervous system, and autonomic nervous system. Reduced respiration rate, heart rate, and blood pressure. These reduce anxiety and stress [19]. The importance of this paper is that by revealing the effect of meditation on heart rate regulation, by increasing awareness and informing the right people, especially heart patients, and taking actions and teaching meditation skills, as well as encouraging people to change their lifestyle, to a large extent It helped improve coronary heart disease and the health of people in the community.

\section{The Effects of Meditation on Heart Rate Variability}

Meditation has positive effects on cardiovascular function. One of the indicators of heart health is heart rate variability (HRV). HRV means the length of time between different heartbeats. If the time interval between heartbeats is longer, the heart is healthier. Changes over time between consecutive heartbeats are mainly dependent on external HR regulation [20,21]. The HRV signal from electrocardiographic signals is strongly correlated with the activity of the autonomic nervous system. HRV represents a dynamic interaction and balance between the sympathetic and parasympathetic systems. The sympathetic system is responsible for raising the heart rate, raising blood pressure, and injecting glucose into the bloodstream to promote activity. In contrast, the parasympathetic nervous system reduces heart rate and nutrient storage, effectively reducing everything [22,23]. HRV analysis is mainly divided into measurements in the time and frequency domain. The pulse intervals' changes to the pulses provided at rest indicate the RR control mechanisms' precise adjustment. Because it helps to assess the balance between sympathetic and parasympathetic effects on heart rhythm. The sympathetic nervous system increases heart rate, which shortens stroke intervals. While the parasympathetic system slows down the heart rhythm, causing longer beats.

The degree of variability in HR provides information about the function of neural control over HR and the heart's ability to respond [24]. HRV reflects the heart's ability to adapt to changing conditions by quickly tracking and responding to unpredictable stimuli. HRV analysis is the ability to assess the overall health of the heart and the state of the autonomic nervous system, which is responsible for regulating heart activity. HRV is a useful signal for studying the autonomic nervous system. HRV refers to changes in pulse intervals and is accountable for balancing the branches of the sympathetic nervous system (SNS) and parasympathetic nervous system (PNS) in the ANS and controlling HR. Normal HR variability is due to autoimmune regulation of the heart and circulatory system [25]. Increased SNS activity or decreased PNS activity leads to increased heart rate. Conversely, a decrease in SNS activity or high PNS activity causes a reduction in heart rate.

\section{The Effect of Meditation on Blood Pressure and Cardiovascular Diseases}

Each year, patients with acute coronary syndrome symptoms and myocardial infarction account for $10 \%$ of emergency cases and up to $25 \%$ of all patient admissions [1,2]. These results represent a significant financial burden on health care resources, with the cost of treating these patients being approximately $\$ 286$ billion annually, or negligibly $17 \%$ of health care costs [3]. He emphasizes that to help solve this problem, cardiovascular interventions such as meditation are needed. Over the past few decades, awareness of the use of meditation or other forms of mind-body therapy in health care has increased.

A meta-analysis of 1264 patients was performed to evaluate cognitive-behavioral therapies (such as biofeedback, relaxation, and meditation) on hypertension [26]. This study showed that cognitive interventions for hypertension are better than no treatment. A meta-analysis reviewed randomized controlled trials and compared blood pressure responses to transcendental meditation (TM) with a control group. This analysis, including 9 randomized controlled trials, included patients from adolescents (mean age 16 years) to the elderly (mean 81 years) [27]. Anderson et al. [28] found that TM reduced systolic blood pressure by an average of $4.7 \mathrm{~mm} \mathrm{Hg}$ and diastolic blood pressure by $3.2 \mathrm{~mm} \mathrm{Hg}$ compared with controls. Subgroup analyses of high blood pressure groups and quality studies have shown similar reductions in blood pressure in all groups. Gregowski et al. [29] evaluated the effect of breathing awareness meditation (BAM) on circulating blood pressure and sodium excretion in African adolescents. They found significant group differences in changes in systolic, diastolic, and nocturnal HR blood pressure over 24 hours and during school hours. Participants in the BAM treatment group showed the most considerable overall reductions in these criteria (Benfroni adjusted $\mathrm{P}<0.05$ ). The researchers concluded that BAM appears to improve hemodynamic function and may affect sodium transfer among African adolescents at increased risk of cardiovascular disease.

Another study also suggested that meditation was associated with beneficial effects on insulin resistance and fat profiles. A survey of 103 people with stable CHD examined the effectiveness of TM on the metabolic CHD syndrome [30]. The primary outcome measures included blood pressure, protein profiles, and insulin resistance. This study showed that the TM group had beneficial changes in regulated systolic blood pressure, insulin resistance, and HRV compared to the control group. However, no significant 
increase in levels of total cholesterol, lipoproteins, and C-reactive protein was observed, which may be due to the high prevalence of statin use and low-density lipoprotein cholesterol levels [30].

Cooper \& Aygen [31] compared 12 patients with hypercholesterolemia with a history of regular meditation practice with 11 individuals in the control group without a history of meditation. They found a $10 \%$ reduction in fasting cholesterol levels in those who meditated over a 13 -month period $(\mathrm{P}<0.005)$. A study published in 2010 evaluated the effects of meditation on 42 volunteers. The participants were divided into two groups: one group participated in one-hour meditation training sessions per week for 5 weeks, and one group formed the control group (normal heart care). After 5 weeks, the meditation group showed a significant decrease in depression and an increase in attendance compared to the control group [32]. Zidane et al. [33] evaluated the benefits of meditation sessions on mental health and cardiovascular variables such as HR and blood pressure. The study included 82 students ( 34 males and 48 females) with no previous meditation experience. They participated in 3 sessions of mindfulness meditation training. This study showed that meditation intervention was effective in reducing heart rate and negative mood, depression, fatigue, and confusion compared to the control group. In 2012, Barnes and Eram-Johnson proposed an updated model of a particular form of meditation [transcendental meditation] [34], which has been shown to reduce the sympathetic nervous system's activation. Dilbeck and
Eram-Johnson reported severe decreases in respiration rate and plasma lactate during TM training, while controls with closed eyes experienced an increase in basal skin resistance. In addition, TM participants had lower baseline HR and respiratory rate, lower plasma lactate levels, and spontaneous reactions of skin resistance outside meditation [35]. Delui et al. [36] examined meditation in patients with cardiovascular disease and depression. Forty-five patients were randomly divided into three groups: relaxation, meditation, and control. After completing the beck depression scale and anxiety self-assessment, patients participated in a series of 10 sessions of Physical Muscle Rest (RMP) or Mental Meditation or received no intervention. Significant reductions in depression, systolic blood pressure, and RH were observed in the meditation group compared with the control group.

\section{Kubious HRV Software}

Kubious software was used to analyze the heartbeat signal before and during meditation. This software is capable of linear and nonlinear analysis of heart signals. The graphical environment of this software is very simple and understandable and can be used easily. This software has the ability to read different formats of ECG signals. In addition to displaying the ECG signal, this software can show R-R intervals at any time by the user. This software is also used to show different time, frequency, and nonlinear analyzes of the cardiac signal. Figure 1 shows the image of Kubious HRV software along with the results of various analyzes.

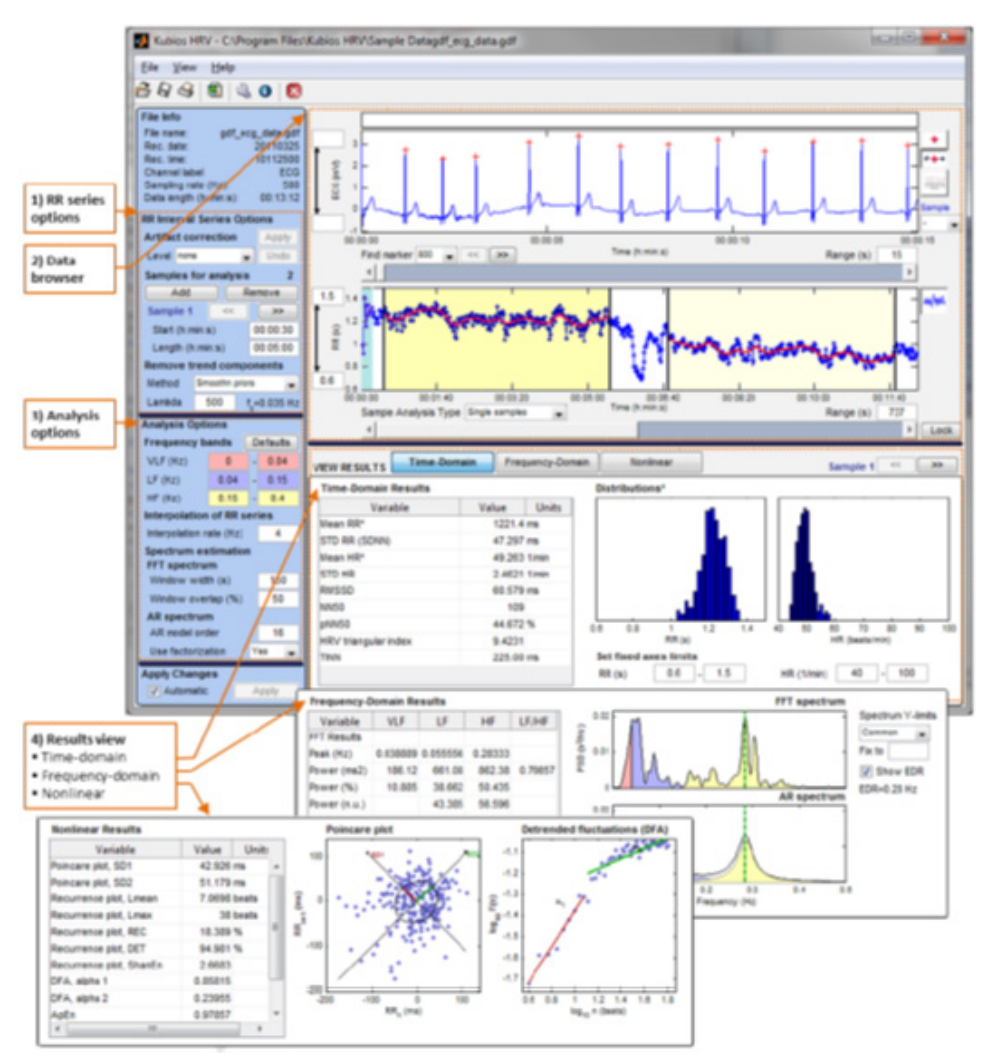

Figure 1: Display the results of cardiac signal analysis before meditation. 
Non- Linear Analysis of Cardiac Signals before and During Meditation

Nowadays, it has been discovered that nonlinear analysis can describe the processes produced by living biological systems. In recent years, nonlinear analysis methods have found many applications in the diagnosis of various conditions. Nonlinear analysis of HRV signal has received a lot of attention for two main reasons. The first reason is the nonlinear nature of the observed signal from the heart, which acts as a nonlinear dynamic oscillator. The second reason is the need to acquire sufficient knowledge about this real phenomenon. Poincaré plot is a nonlinear method for extracting hidden information from the cardiac signal. In this method, each beat is considered in terms of the next beat and an elliptical shape is obtained that has short-term (SD1) and long-term changes (SD2) [37]. Calculating the parameters SD1 and SD2 in the two previous states and during meditation using Kubius software are given in Tables $1 \& 2$.

Table 1 shows the nonlinear parameters' values before meditation, and Table 2 shows the amounts of nonlinear parameters during meditation. As the nonlinear parameters' values show, when the person is during meditation, the heart signal is calmer and fluctuates less. So meditation can regulate your heart rate. Figure 2 shows the changes in features introduced in the two states before and during meditation.
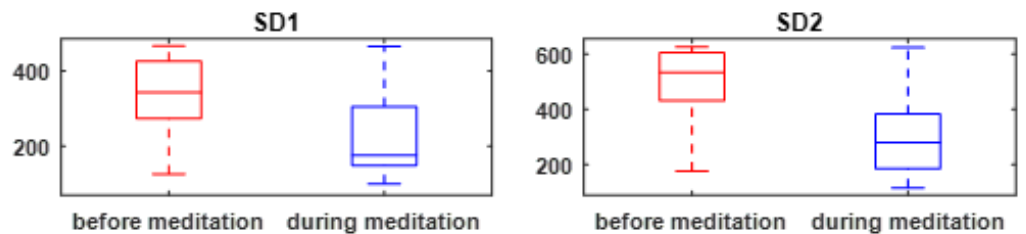

SD1/SD2
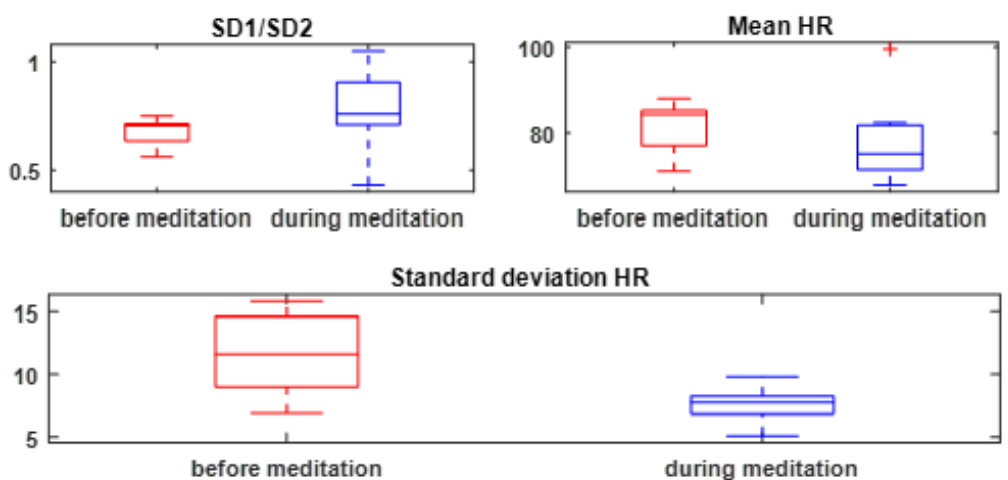

Figure 2: Boxplots of the most significant linear and nonlinear indices before and during meditation.

Table 1: Nonlinear features extracted from the cardiac signal before meditation.

\begin{tabular}{|c|c|c|c|c|c|c|c|c|c|}
\hline Feature type & C1 & C2 & C3 & C4 & C5 & C6 & C7 & C8 & Mean \pm std \\
\hline SD1 (ms) & 125.8 & 434.1 & 372.7 & 308.2 & 418.2 & 466.6 & 312.4 & 238.2 & $334.52 \pm 113.29$ \\
\hline SD2 (ms) & 175.6 & 609.7 & 572.9 & 493.3 & 598.7 & 624.9 & 442.9 & 418.5 & $492.06 \pm 149.94$ \\
\hline SD1/SD2 & 0.72 & 0.71 & 0.65 & 0.62 & 0.7 & 0.75 & 0.71 & 0.56 & $0.68 \pm 0.06$ \\
\hline Mean HR & 71.03 & 83.99 & 85.25 & 84.61 & 77.45 & 76.65 & 85.22 & 87.99 & $81.52 \pm 5.80$ \\
\hline STD HR & 10.09 & 14 & 15.27 & 15.83 & 6.89 & 7.9 & 12.37 & 10.88 & $11.65 \pm 3.30$ \\
\hline
\end{tabular}

Table 2: Nonlinear features extracted from the cardiac signal during meditation.

\begin{tabular}{|c|c|c|c|c|c|c|c|c|c|}
\hline Feature type & C1 & C2 & C3 & C4 & C5 & C6 & C7 & C8 & Mean \pm std \\
\hline SD1 (ms) & 145.4 & 465.5 & 325.3 & 99.4 & 152.4 & 184.1 & 165.9 & 284.8 & $227.85 \pm 121.86$ \\
\hline SD2 (ms) & 152.8 & 623 & 309.6 & 114.6 & 353 & 251.3 & 216.3 & 411.5 & $304.01 \pm 162.54$ \\
\hline SD1/SD2 & 0.95 & 0.75 & 1.05 & 0.86 & 0.43 & 0.73 & 0.77 & 0.69 & $0.78 \pm 0.19$ \\
\hline Mean HR & 73.75 & 82.4 & 74.76 & 75.44 & 67.83 & 69.12 & 81.21 & 99.59 & $78.01 \pm 10.10$ \\
\hline STD HR & 8.07 & 6.32 & 7.66 & 9.81 & 5.11 & 7.85 & 7.37 & 8.43 & $7.58 \pm 1.40$ \\
\hline
\end{tabular}




\section{Discussion and Conclusion}

Nowadays, anxiety is considered the most common psychiatric disorder, and various medical and non-pharmacological techniques and methods are used to relieve it and its stress. One of the most useful and common treatments for anxiety is meditation. Meditation is generally a technique of mastering the mind. According to the proponents of this method, the goal of meditation is to train and control the mind by introspective techniques to achieve the joy of deep and long-lasting awareness and peace, independent of others. Meditation means focusing on the mind. This focus can be on a voice, phrase or prayer, object, image, self-awareness, how to breathe, religious duties, and so on. The goal is to be aware of the moment, achieve relaxation, and reduce stress. Meditation promotes personality and spiritual growth [38]. It does not matter which meditation method you use because their effects on the body are similar. Researchers have shown that meditation reduces stress hormone levels and contributes to the health of the immune system. Meditation has been shown to lower cholesterol levels in the blood and help keep arteries open by reducing hormones such as Epinephrine. Also, studies based on AJ have demonstrated that this technique increases alpha waves' intensity, which indicates a state of relaxation and acceptance, to an even higher level than sleep time [39]. Research has also shown that improving blood flow, which protects the arteries, lowers blood lactic acid levels, which is associated with reduced anxiety and reduces heart rate, which reduces the need for pumping, giving the heart a chance to rest. Another effect of meditation is slow breathing, as a result of which the body consumes less oxygen.

Many studies have examined the effect of meditation on heart health. Regular meditation practice has been shown to help lower high blood pressure in the long run. Among these studies, one study showed a sharp drop in blood pressure and heart rate in black adults. Another study found that teens who meditate twice a day for 15 minutes each time for four months can lower their blood pressure up to several digits [40]. The sympathetic nervous system dilates the pupil and raises blood pressure and respiration rate. Meditation works the other way around, lowering blood pressure and muscle tension. This article shows that meditation is an influential factor in reducing heart rate, increasing concentration, and reducing anxiety and stress. Therefore, since meditation is a simple and low-cost method, meditation exercises can be one of the most common problems for people and Reduced heart disease. As a result, using meditation by regulating heart rate reduces mental fatigue and improves performance.

\section{Funding Statement}

The study received no funding.

\section{Conflict of Interest}

The authors declare no conflict of interest.

\section{Ethics}

Not applicable.

\section{References}

1. Niska R, Bhuiya F, Xu J (2010) National Hospital Ambulatory Medical Care Survey: 2007 emergency department summary. Natl Health Stat Report (26): 1-31.

2. Pinkstaff S, Peberdy MA, Kontos MC, Finucane S, Lavie CJ, et al. (2011) The influence of referral source on characteristics of patients undergoing cardiac stress testing: emergency department versus outpatient office. Postgrad Med 123(2): 36-41.

3. Menezes AR, Lavie CJ, Milani RV, O’Keefe J, Lavie TJ (2011) Psychological risk factors and cardiovascular disease: is it all in your head? Postgrad Med. 123(5): 165-176.

4. Clougherty JE, Kubzansky LD (2009) A framework for examining social stress and susceptibility to air pollution in respiratory health. Environ Health Perspect 117(9): 1351-1358.

5. Lichtman JH, Bigger JT Jr, Blumenthal JA, Frasure-Smith N, Kaufmann PG, et al. (2008) Depression and coronary heart disease. Recommendations for screening, referral, and treatment. A science advisory from the American Heart Association Prevention Committee to the Council on Cardiovascular Nursing, Council on Clinical Cardiology, Council on Epidemiology and Prevention, and Interdisciplinary Council on Quality of Care Outcomes Research. Circulation 118(17): 1768-1775.

6. Rozanski A, Blumenthal JA, Kaplan J (1999) Impact of psychological factors on the pathogenesis of cardiovascular disease and implications for therapy. Circulation 99(16): 2192-2217.

7. Musselman DL, Tomer A, Manatunga AK, Knight BT, Porter MR, et al. (1996) Exaggerated platelet reactivity in major depression. Am J Psychiatry 153(10): 1313-1317.

8. Laghrissi-Thode F, Wagner WR, Pollock BG, Johnson PC, Finkel MS (1997) Elevated platelet factor 4 and beta-thromboglobulin plasma levels in depressed patients with ischemic heart disease. Biol Psychiatry 42(4): 290-295.

9. Gaab J, Blatter N, Menzi T, Pabst B, Stoyer S, et al. (2003) Randomized controlled evaluation of effects of cognitive- behavioral stress management on cortisol responses to acute stress in healthy subjects. Psychoneuo endocrinology 28(6): 767-779.

10. Akbari M, Mahmood Aliloo M, Aslanabadi N (2010) Relationship between Stress and Coping Styles with Coronary Heart Disease: Role of Gender Factor. Iran J Psychiatry Clin Psychol 5(4): 368-76.

11. Yeung AC, Vekshtein VI, Krantz DS, Vita JA, Ryan TJ, et al. (1991) The Effect of atherosclerosis on the vasomotor response of the coronary arteries to mental stress. N Engl J Med 325(22): 1551-1556.

12. Suarez EC (2003) Plasma interleukin-6 is associated with psychological coronary risk factors: moderation by use of multivitamin supplements. Brain Behav Immun 17(4): 296-303.

13. Boltwood MD, Taylor CB, Burke MB, Grogin H, Giacomini J (1993) Anger report predicts coronary artery vasomotor response to mental stress in atherosclerotic segments. Am J Cardiol 72(18): 1361-1365.

14. Markovitz JH (1998) Hostility is associated with increased platelet activation in coronary heart disease. Psychosom Med 60(5): 586-591.

15. Saeed SA, Antonacci DJ, Bloch RM (2010) Exercise, yoga, and meditation for depressive and anxiety disorders. Am Fam Physician 81(8): 981-986.

16. Mathew KL, Whitford HS, Kenny MA, Denson LA (2010) The long-term effects of mindfulness-based cognitive therapy as a relapse prevention treatment for major depressive disorder. Behav Cogn Psychother 38(5): 561-576. 
17. Goldin PR, Gross JJ (2010) Effects of mindfulness-based stress reduction (MBSR) on emotion regulation in social anxiety disorder. Emotion 10(1): 83-91.

18. Lazar SW, Bush G, Gollub RL, Fricchione GL, Khalsa G, et al. (2000) Functional brain mapping of the relaxation response and meditation. Neuroreport 11(7): 1581-1585.

19. Phongsuphap S, Pongsupap Y (2011) Analysis of Heart rate variability during meditation by a pattern recognition method. Computing in cardiology 38: 197-200.

20. www.ncnm.edu/helfgottresearch/projects/mind-body-machine/meditation-and

21. Manocha R, Gordon A, Black D, Malhi G (2009) Using meditation for less stress and better wellbeing A seminar for GPs. Australian Family Physician 38(6): 454-458.

22. Manish Yadav, Wadhwani AK (2011) Filtration of ECG signal by Using Various Filter. International Journal of Modern Engineering Research 1(2): 658-661.

\section{WWW.PHYSIONET.ORG/TUTORIALS/HRVTOOLKIT}

24. Hegde V, Hegde R, Satyanarayana PS (2012) Meditation: its tremendous impact on heart rate variability.

25. Eisenberg DM, Delbanco TL, Berkey CS, Kaptchuk TJ, Kupelnick B, et al. (1993) Cognitive behavioral techniques for hypertension: are they effective? Ann Intern Med 118(12): 964-972.

26. Anderson JW, Liu C, Kryscio RJ (2008) Blood pressure response to transcendental meditation: a meta-analysis. Am J Hypertens 21(3): 310-316.

27. Rainforth MV, Schneider RH, Nidich SI, Gaylord-King C, Salerno JW, et al. (2007) Stress reduction programs in patients with elevated blood pressure: a systematic review and meta-analysis. Curr Hypertens Rep 9(6): 520-528.

28. Gregoski MJ, Barnes VA, Tingen MS, Harshfield GA, Treiber FA (2011) Breathing awareness meditation and LifeSkills Training programs influence upon ambulatory blood pressure and sodium excretion among African American adolescents. J Adolesc Health 48(1): 59-64.

29. Paul-Labrador M, Polk D, Dwyer JH, Velasquez I, Nidich S, et al. (2006) Effects of a randomized controlled trial of transcendental meditation on components of the metabolic syndrome in subjects with coronary heart disease. Arch Intern Med 166(11): 1218-1224.
30. Cooper MJ, Aygen MM (1979) A relaxation technique in the management of hypercholesterolemia. J Human Stress 5(4): 24-27.

31. Leite JR, Ornellas FL, Amemiya TM, de Almeida AAF, Aparecido Dias A, et al. (2010) Effect of progressive self-focus meditation on attention, anxiety, and depression scores. Percept Mot Skills 110 (3 Pt 1): 840-848.

32. Zeidan F, Johnson SK, Gordon NS, Goolkasian P (2010) Effects of brief and sham mindfulness meditation on mood and cardiovascular variables. J Altern Complement Med 16(8): 867-873.

33. Barnes VA, Orme-Johnson DW (2012) Prevention and treatment of cardiovascular disease in adolescents and adults through the Transcendental Meditationt program: a research review update. Curr Hypertens Rev 8(3): 227-242.

34. Dillbeck MC, Orme-Johnson DW (1987) Physiological differences between Transcendental Meditation and rest. Am Psychol 42(9): 879-881.

35. Delui MH, Yari M, Khouyinezhad G, Amini M, Bayazi MH (2013) Comparison of cardiac rehabilitation programs combined with relaxation and meditation techniques on reduction of depression and anxiety of cardiovascular patients. Open Cardiovasc Med J 7: 99-103.

36. Karimi Moridani M, Marjani S (2020) A Review of the Methods for Sudden Cardiac Death Detection: A Guide for Emergency Physicians. International Association of Online Engineering 16(9): 137-158.

37. Jain S, Shapiro SL, Swanick S, Roesch SC, Mills PJ, et al. (2007) A randomized controlled trial of mindfulness meditation versusrelaxation training: Effects on distress, positive stress of mind, rumination, and distraction. Annals of Behavioral Medicine 33(1): 11-21.

38. Lee SH, Ahn SC, Lee YJ, Choi TK, Yook KH, et al. (2007) Effectiveness of a meditation-based stress management program as an adjunct to pharmacotherapy in patients with anxiety disorder. Journal of Psychosomatic Research 62(2): 189-195.

39. Paul G, Elam B, Verhulst SJ (2007) A longitudinal study of student's perceptions of using deep breathing meditation to reduce testing stress. Teaching and learning in Medicine 19(3): 287-292.

40. Paholpak S, Piyavhatkul N, Rangseekajee P, Krisanaprakornkit T, Arunpongpaisal S, et al. (2012) Breathing meditation by medical students at Khon Kaen University: effect on psychiatric symptoms, memory, intelligence and academic achievement. J Med Assoc Thai 95(3): 461-469. 